\title{
Zerbrochene Marknagelung - Tipps und Tricks für den komplizierten Fall
}

\author{
Roland Biber, Hermann Josef Bail
}

\section{Zusammenfassung}

Zum Bruch eines Marknagels kommt es insbesondere bei verzögerter Frakturheilung, Pseudarthrose und persistierenden Knochendefekten. Betroffen sind meist charakteristische Schwachstellen: Proximale Femurnägel brechen häufig durch die große Nagelöffnung, wo der Nagel das Tragelement aufnimmt. Der typische Tibianagelbruch ist dagegen distal im Bereich eines Verriegelungslochs lokalisiert. Insgesamt sind Nagelbrüche selten, und die Bewertung der Vorgehensweisen zur Entfernung der Implantatfragmente basiert überwiegend auf Einzelfallbeschreibungen und kleinen Fallserien. Technisch ist die Entfernung beschädigter Verriegelungsnägel sehr variationsreich: Die Entfernung von meist markraumausfüllenden Hohlnagelfragmenten erfolgt mit gänzlich anderen Instrumenten als die Entfernung der oft weniger dicken Reste solider Nägel. Wiederum andere Methoden erfordert die Entfernung eines nicht vollständig gebrochenen, jedoch verbogenen Marknagels. Neben dem Marknagel selbst können auch andere Bestandteile der Nagelosteosynthese brechen, etwa Verriegelungsschrauben oder Tragelemente eines proximalen Femurnagels. Kommt es ferner zu Beschädigungen der Hexagonstruktur eines Inbus, so sollten zum Ausdrehen der betroffenen Komponente spezielle Hilfsmittel zur Verfügung stehen. Bei der Entfernung eines gebrochenen Marknagels sollte der Operateur auf alle diese Eventualitäten vorbereitet sein. Dies schließt die Kenntnis der in diesem Beitrag vorgestellten Methoden, aber auch das Vorhandensein der notwendigen Spezialinstrumentarien ein, die inzwischen kommerziell erhältlich sind.

\section{Broken Intramedullary Nail Removal - Tips and Tricks for Difficult Cases}

The reasons for intramedullary nail breakage include delayed union, nonunion, and chronic instability due to persistent bone defects. The affected sites appear to be characteristic zones of weakness: Proximal femoral nail breakage typically occurs through the nail's proximal aperture, where the cervical head screw is fixed. However, the typical site for tibial nail fracture is around a distal interlocking hole. Nail breakage is rare, which is why evidence on treatment methods is mainly based on case reports and small case series. There are many technical variations for the removal of damaged intramedullary nails: Extracting large hollow nail fragments is a completely different procedure from extracting the remnant of a small caliber solid nail. Other methods are necessary if a nail is bent but not completely broken. Beside the nail, other components of osteosynthesis may break, i.e. interlocking screws or cervical lag screw elements of proximal femoral nails. Damaged hexagons may further impede the removal of certain components without specific aids. The operator should be prepared for such problems when removing broken intramedullary nails. Preparation includes knowledge of the methods presented in this article, as well as the availability of specific instruments for broken implant removal, which are now offered by several manufacturers.

\section{Einleitung}

Die Notwendigkeit der Entfernung intakter Marknägel nach knöcherner Heilung hat schon Gerhard Küntscher infrage gestellt [7]. Trotz der damit verbundenen Risiken werden Metallentfernungen häufig ohne zwingende Indikation auf Patientenwunsch hin durchgeführt $[5,6]$. Bereits die Entfernung intakter Nägel kann problematisch sein, etwa wenn Nageleintrittsstelle oder Verriegelungsschrauben knöchern überbaut sind, Kno-

OP-JOURNAL 2016; 32: 146-150

(c) Georg Thieme Verlag KG Stuttgart · New York DOI http://dx.doi.org/10.1055/s-0042-106932 chen in das Implantat eingewachsen ist oder Implantatverbindungen sich nicht lösen lassen.

Das Versagen einer Marknagelosteosynthese - insbesondere der Nagelbruch ist häufig das Resultat einer Instabilität bei unzureichender Knochenheilung (Pseudarthrose, verbliebene Defekte) und damit eine zwingende Indikation zur Implantatentfernung.

In der Literatur gibt es bisher relativ wenige systematische Arbeiten zum Thema der komplizierten Marknagelentfernung, überwiegend sind einzelne Aspekte und technische Hinweise kasuistisch dargestellt $[2,10]$.
In der vorliegenden Arbeit wird versucht, die möglichen Probleme darzustellen, die sich aufgrund Implantatversagens bei der Marknagelentfernung ergeben können. So kann neben dem Bruch oder der Verbiegung des Nagels auch der Bruch von Verriegelungsschrauben oder von Tragelementen (z.B. proximaler Femurnagel) vorliegen. Ferner können Gewinde festgelaufen oder Inbusverbindungen hohlgedreht sein. Auf die Vielzahl dieser denkbaren Probleme sollte der Operateur bei der Durchführung einer Metallentfernung eingestellt sein. Auch in einem Übersichtsartikel ist es jedoch nicht möglich, alle denkbaren technischen Varianten vollständig wiederzugeben. Die nachfolgende Dar- 


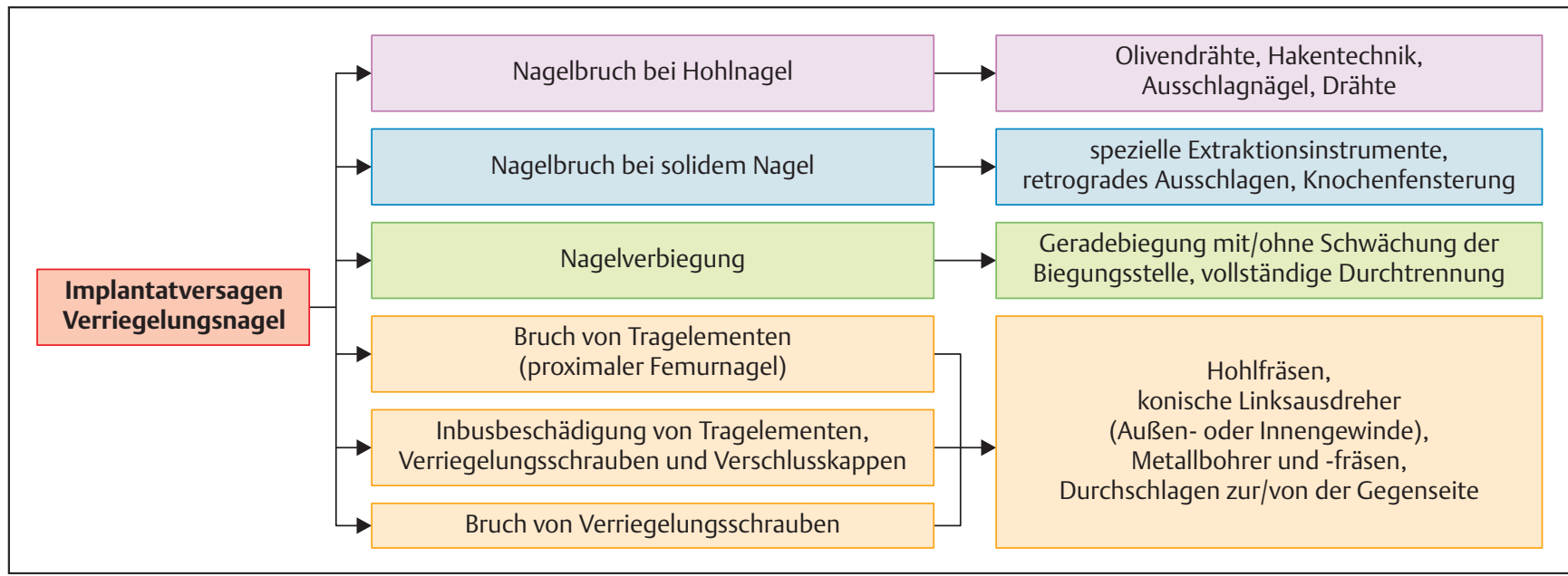

Abb. 1 Typische Probleme und Lösungsoptionen bei der Entfernung defekter Marknägel.

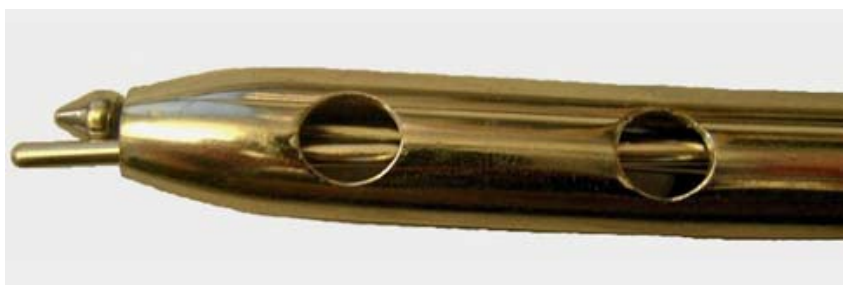

Abb. 2 Die Verklemmung eines Olivendrahts an der Nagelspitze ermöglicht das Ausschlagen kanülierter Nägel ohne weiteres Spezialinstrumentarium. stellung spiegelt demgemäß die von den Autoren bevorzugten Methoden wider.

\section{Implantatversagen bei Marknägeln}

Der Bruch eines Marknagels ist ein seltenes Ereignis und kommt praktisch ausschließlich bei chronischer Überlast des Nagels vor, etwa bei Knochendefekten oder Pseudarthrosen. Für Femurnägel wird die Prävalenz mit 0,2-5,7\% eingeschätzt [2]. Für die Tibia lässt sich aus den vorliegenden Fallberichten keine Prävalenz abschätzen, jedoch scheint es, dass Nagelbrüche hauptsächlich nach distalen Frakturen und im Bereich eines distalen Verriegelungslochs auftreten [4]. Die Inzidenz von Brüchen der Verriegelungsschrauben wird zwischen 2 und $41 \%$ eingeschätzt [8].

Bei der Entfernung gebrochener Marknägel können verschiedene zusätzliche Probleme einzeln oder in Kombination vorliegen, die mitunter präoperativ nicht eingeschätzt werden können.

So ist der Nagelbruch selbst i.d.R. durch eine adäquate bildgebende Diagnostik (konventionelles Röntgen in 2 Ebenen, Computertomografie) meist erkennbar; für einen hohlgedrehten Inbus oder den Bruch von Verriegelungsschrauben nahe an einem Nagelloch gilt das dagegen nicht unbedingt.
Mehrere Publikationen weisen auf die Risiken und Gefahren bereits bei vermeintlich unkomplizierten Materialentfernungen hin [6]. Um so mehr sollte der Operateur bei der Entfernung eines schadhaften Implantats auf alle Eventualitäten mental und technisch und vorbereitet sein. Dies beinhaltet die genaue Kenntnis des zu entfernenden Implantats einschließlich des Durchmessers der möglicherweise schadhaften Komponenten. Neben den zugehörigen Standardentfernungssieben sollten Spezialsets für problematische Materialentfernungen zur Verfügung stehen, wie sie inzwischen von mehreren Herstellern angeboten werden. Abb. 1 gibt einen Überblick über das Armamentarium für die verschiedenen Problemstellungen.

\section{Gebrochene kanülierte Marknägel}

Die ursprünglichen Küntscher-Nägel waren geschlitzte Hohlnägel, sodass für die Entfernung von Nagelfragmenten dieser Art die längste Erfahrung besteht. Entsprechend bietet die Literatur eine ganze Reihe von Vorschlägen [9]:

- Direkte Bergung mit kräftigen Rongeuren oder laparoskopischen Fasszangen.

- Impaktierung eines kleineren Nagels in das (noch durch Verriegelungsschrauben fixierte) Fragment des zu entfernenden Hohlnagels: Gelingt eine entsprechende Verklemmung ineinander, so kann dann die Extraktion beider Teile en bloc erfolgen.

- Impaktierung von starren Bohrern, konischen Gewindeinstrumenten oder dünnen Marknägeln in das Nagelfragmentinnere zwecks Extraktion.

- Einsatz hakenförmiger Instrumente mit dem Ziel des Einhakens in Verriegelungslöcher oder an der Nagelspitze.

- Einführen eines Drahtes in das Nagelinnere und Ausleiten des Drahtes durch ein Verrieglungsloch mittels einer weiteren, zuvor durch das Verriegelungsloch eingeführten Drahtschinge. Nach Verzwirbeln dieses Drahtes kann dieser nicht mehr durch das Verriegelungsloch zurückgleiten, sodass bei einem Herausziehen des Drahtes das abgebrochene Nagelfragment extrahiert wird.

- Oliventechnik (Abb.2): Ein am Ende mit einer Olive versehener Führungsdraht wird durch das zu bergende kanülierte Nagelfragment hindurch und ein Stück weit darüber hinaus vorgeschoben. Weitere Führungsdrähte werden dann ebenfalls komplett durch das Nagelfragment hindurch geschoben, wodurch das Loch im Bereich der Nagelspitze verengt wird. Beim Zurückziehen des Olivendrahts kann dieser die Kanülierung des Nagels nicht mehr passieren und nimmt das zu entfernende Nagelfragment mit.

\section{Gebrochene solide Marknägel}

Für die Entfernung solider Nagelfragmente eignen sich die für Hohlnägel beschriebenen Techniken nicht, sodass an sich ausgedehntere chirurgische Maßnahmen angezeigt scheinen [3]. Die erste beschriebene Extraktionstechnik wirkt dabei aus heutiger Sicht sehr behelfs- 
mäßig: Hellemondt und Haeff beschrieben 1996 die Entfernung eines $8 \mathrm{~mm}$ durchmessenden soliden Nagels durch Einschlagen eines größeren Hohlnagels über das (anfangs noch verriegelte) solide Nagelfragment hinweg. Natürlich kommt eine solche Technik ausschließlich für relativ dünne Marknägel bzw. Nagelbrüche im Bereich größerer Markraumdurchmesser infrage. Entsprechend wurden weitere Methoden publiziert, etwa das Durchschlagen des Nagelfragments nach distal oder das Ausschlagen nach proximal durch einen distalen $\mathrm{Ar}$ beitszugang oder eine Knochenfensterung.

Zwischenzeitlich bieten jedoch mehrere Hersteller spezielle Instrumentarien für die Entfernung abgebrochener solider Nagelfragmente an (Abb. 3)

Das Grundprinzip ist dabei eine Art Becher mit einem konischen Innengewinde, welches über das verriegelte oder anderweitig fixierte Nagelfragment geführt wird und mit diesem verklemmt (Abb. 4). Fakultativ kann das Fragment zuvor vorsichtig mit einer Hohlfräse teilweise überbohrt und so etwas gelöst werden. Auch ein vorheriges Aufbohren der Markraumenge kann erforderlich sein, um mit dem Extraktionsinstrument der erforderlichen Größe passieren zu können.

\section{Entfernung verbogener Marknägel}

Durch ein erneutes Trauma kann es zur Verbiegung eines einliegenden Marknagels kommen, ohne dass dieser vollständig durchbricht. Die Extraktion eines solchen Implantats stellt dann eine besondere Herausforderung dar, da ein gewaltsames Ausschlagen des verbogenen Nagels zu weiteren Knochenschäden führen kann.

Mehrere Techniken zur Entfernung verbogener Marknägel sind kasuistisch beschrieben. Die Grundidee ist dabei entweder die Geradebiegung des Nagels oder seine komplette Durchtrennung an der Biegungsstelle mit nachfolgender Bergung der Fragmente $[1,10]$. Das Geradebiegen kann dabei u.U. geschlossen erfolgen, wobei evtl. eine sehr starke Gewalteinwirkung mit der Gefahr zusätzlicher Weichteilschäden erforderlich ist. Erleichtert werden kann das Manöver durch eine gezielte Schwächung des Nagels an der Biegungsstelle, etwa durch offenes oder perkutanes Anbohren [1] oder durch den Einsatz spezieller Trennschleifer.

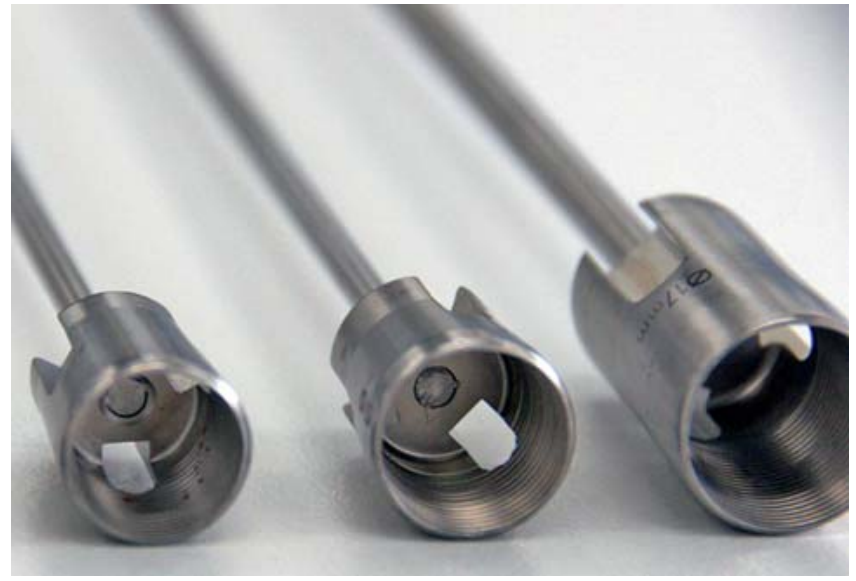

Abb. 3 Instrumentarium zur Extraktion solider Marknagelfragmente.

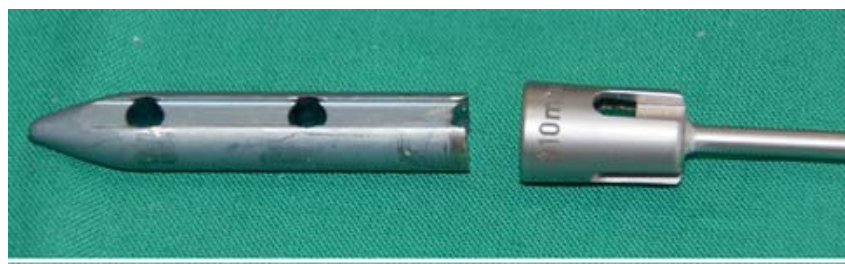

Abb. 4 Entfernung eines soliden Nagelfragments mittels eines Extraktionsinstrumentariums. Ein konisches Innengewinde passender Größe wird dabei mit dem noch verriegelten Nagelfragment verbunden. Nach Entriegelung kann das abgebrochene Teil herausgezogen werden.

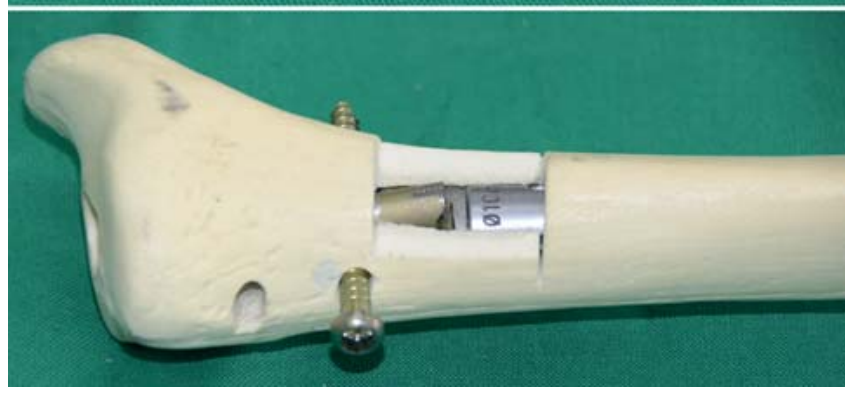

Im Falle der beabsichtigten oder versehentlichen vollständigen Durchtrennung des verbogenen Nagels kann die Entfernung analog dem Vorgehen bei gebrochenen Marknägeln durchgeführt werden. Nach offener Durchtrennung des Nagels an der Biegungsstelle ist darüber hinaus die Bergung entweder beider Nagelfragmente oder nur des distalen Nagelfragments über die Frakturstelle beschrieben [10].

\section{Inbusbeschädigung von Tragelemen- ten, Verriegelungsschrauben und Verschlusskappen}

Eine besondere Problematik stellt das Implantatversagen bei proximalen $\mathrm{Fe}$ murnägeln dar [2]. Cruz-Sánchez et al. fanden 11 Nagelbrüche auf 1246 Frak- turversorgungen $(0,88 \%)$ und identifizierten dabei 3 Typen [2]:

- Nagelbruch durch die proximale Nagelöffnung (64\%)

- Bruch des Tragelements/der Tragschraube (27\%)

- Bruch durch ein distales Nagelverriegelungsloch (9\%)

Durch übermäßig festes Andrehen im Rahmen der Erstimplantation, aber auch durch das Einwachsen von Knochen unterhalb eines nicht rückschneidenden Gewindes kann es darüber hinaus zum Festsitzen von Tragelementen kommen.

Der Versuch des Ausdrehens im Rahmen der Metallentfernung kann dann wiederum zum Hohldrehen von Inbusverbindungen oder zum Abbrechen von Schraubendrehern führen. 


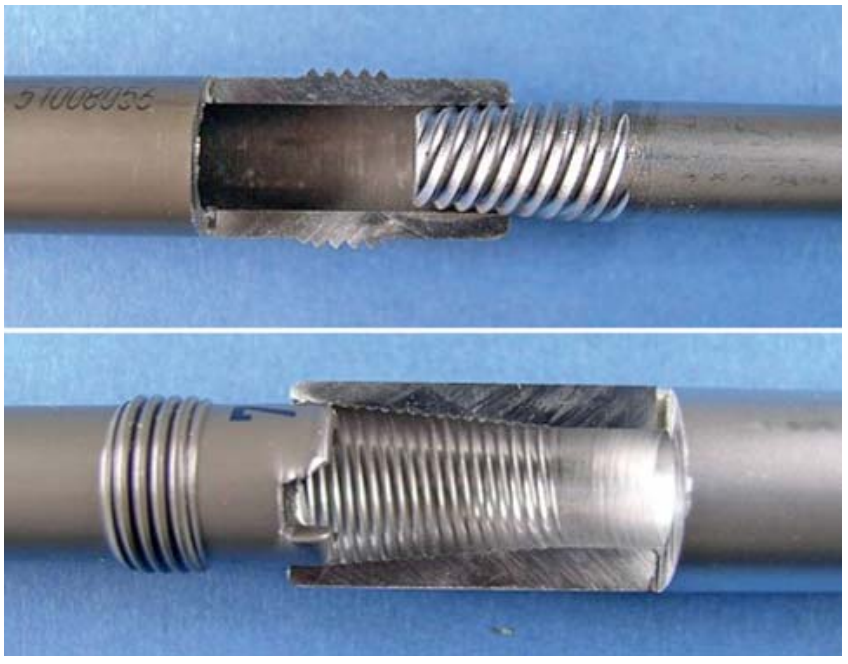

Zwei Arten von Extraktionsinstrumenten bieten sich für derartige Problemfälle an (Abb. 5):

1. konische linksdrehende Außengewinde verschiedener Größe

2 . konische linksdrehende Innengewinde verschiedener Größe

Zusätzlich sollte ein Set mit Hohlfräsen verschiedener Durchmesser zur Verfügung stehen. Ein wichtiger Schritt zur Befreiung festsitzender Tragelemente ist das vorherige vorsichtige Überfräsen der von Knochen umgebenen Abschnitte des Implantatfragments. Zur Bergung abgebrochener Tragelemente in Schenkelhals und Femurkopf sind solche Hohlfräsen häufig bereits ausreichend: Unter Bildwandlerkontrolle in 2 Ebenen können diese Implantatfragmente vorsichtig überfräst werden, wobei darauf geachtet werden muss, dass sie nicht in das Azetabulum vorgetrieben werden. Nach vollständiger Überfräsung ist mit dem Zurückziehen der Fräse auch das Implantatfragment geborgen.
Abb. 5 Linksdrehende konische Außen- und Innengewinde.

Schwieriger gestaltet sich häufig die Entfernung von Tragelementen oder Verschlusskappen, die im Nagel festsitzen und deren Inbus beschädigt oder hohlgedreht ist. Eigene, in Kooperation mit der Firma Aesculap durchgeführte Versuche haben gezeigt, dass ein Verklemmen oder Festfressen von Tragelementen im Nagel wahrscheinlich nicht stattfindet: Auch unter vertikaler Belastung des Tragelements mit $550 \mathrm{~N}$ konnte eine mit einem üblichen Drehmoment von etwa 20 Nm eingebrachte Traghülse unter Anwendung eines entgegengesetzten Drehmoments von ca. $15 \mathrm{Nm}$ wieder ausgedreht werden.

Dennoch kann es bei reduzierten Angriffsmöglichkeiten schwierig sein, dieses für die Entfernung notwendige Drehmoment auf die betroffene Implantatkomponente zu übertragen.

Wichtig ist auch hier das vorsichtige Überfräsen mittels Hohlfräse soweit wie möglich. Hierdurch wird einerseits Knochen entfernt, der die Rückwärtsbewe- gung der Implantatkomponente behindern könnte. Andererseits wird Platz für den Ansatz eines Innengewindeinstruments geschaffen. Mit einem gut aufgesetzten Linksausdreher können dann die benötigten Drehmomente aufgebracht werden (Abb. 6).

Für die Entfernung von hohlgedrehten Verriegelungsschrauben kommen ebenfalls die beschriebenen Linksausdreher in Betracht, wobei auch hier vorher eventuell eine kleine Hohlfräse angewendet werden sollte, um störenden Knochen kontrolliert und sparsam zu beseitigen. Der Zustand des Schraubenkopfs bestimmt dann, ob besser ein Außen- oder Innengewinde angesetzt werden kann. Ist der Schraubenkopf zu stark beschädigt, kann er ggf. abgezwickt oder weggebohrt werden. Nach dosiertem Überfräsen kann dann das verbliebene Gewinde mittels eines kleineren linksdrehenden Innengewindes entfernt werden (Abb. 7).

\section{Gebrochene Verriegelungsschrauben}

Die häufigsten Gründe für den Bruch von Verriegelungsschrauben sind Überlast bei Trümmerfrakturen, verzögerte Knochenheilung und Pseudarthrose, erneutes Trauma und Incompliance des Patienten [8]. Es gilt also, neben der gebrochenen Schraube auch das zugrunde liegende Problem zu analysieren und anzugehen. Hier kann es etwa sinnvoll sein, einen Nagelwechsel mit Aufbohrung der Markhöhle durchzuführen.

Die Entfernung des Schraubenkopfs erfolgt in üblicher Weise durch Ausdrehen mit einem Schraubendreher, bei Inbusproblemen können die oben beschriebenen Methoden zum Einsatz kommen. Das verbliebene Schraubenspitzenfragment behindert in vielen Fällen die Na-
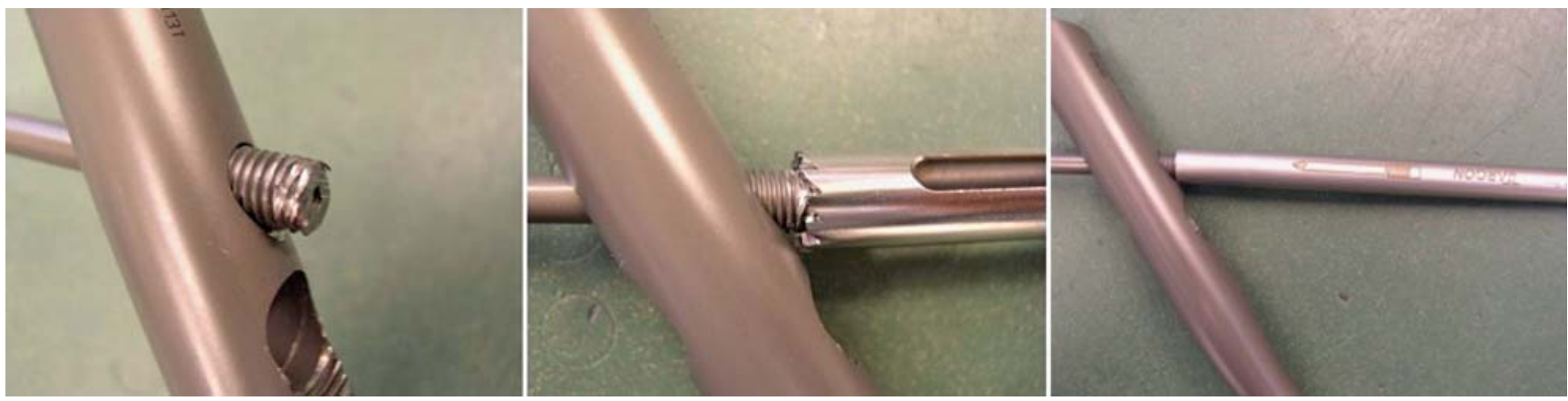

Abb. 6 Entfernung eines beschädigten Tragelements am Beispiel eines Antirotations-Pins des Targon PFT-Nagels. Nach Abbruch des gesamten Inbus ist ein Ausdrehen mit dem Hexagonschraubendreher nicht mehr möglich (links). Durch Überbohren mit einer passenden Hohlfräse wird der umgebende Knochen sparsam, aber vollständig entfernt (Mitte). Dies ermöglicht das Aufsetzen eines konischen linksdrehenden Innengewindeinstruments, mit dem hohe Drehmomente aufgebracht und der beschädigte Antirotations-Pin ausgedreht werden kann (rechts). 

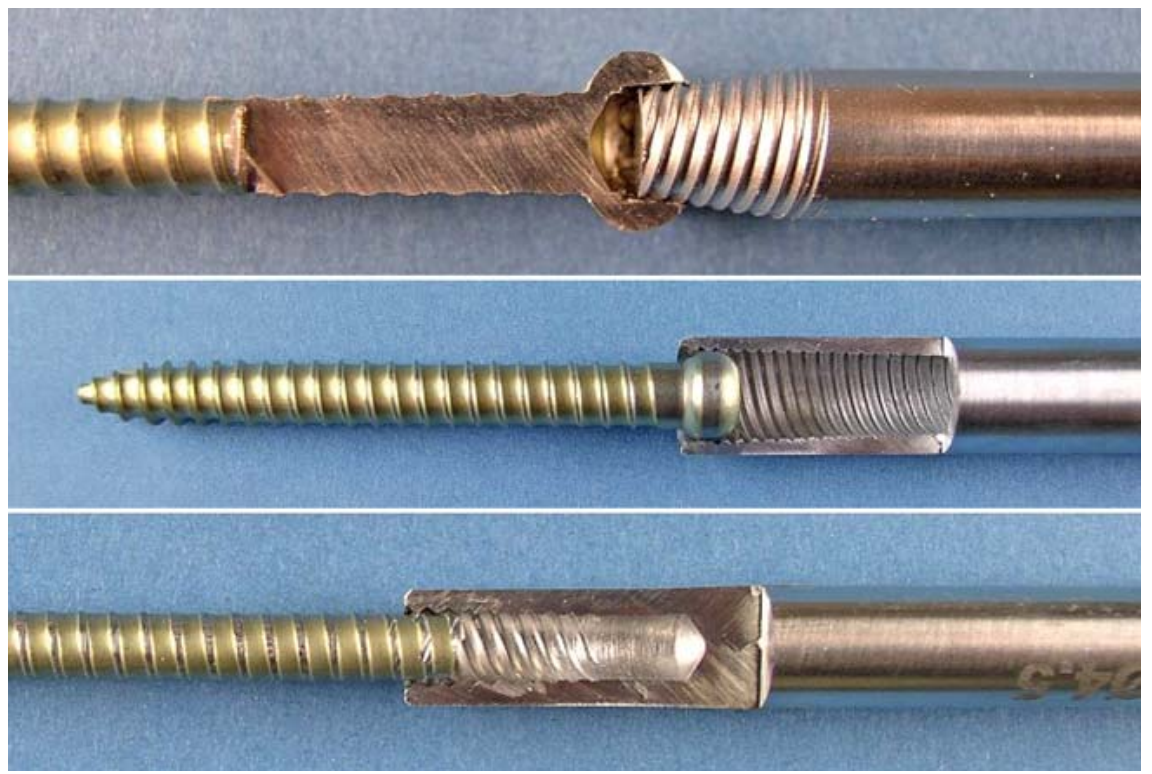

Abb. 7 Verwendung von Linksausdrehern zur Entfernung beschädigter Verriegelungsschrauben. Bei vorhandenem Schraubenkopf kann ein konisches Außen- oder Innengewinde zum Einsatz kommen (oben, Mitte). Ist der Schraubenkopf nicht mehr vorhanden oder muss er weggebohrt werden, so kann nach vorsichtigem Überfräsen ein kleineres Außengewinde verwendet werden (unten).

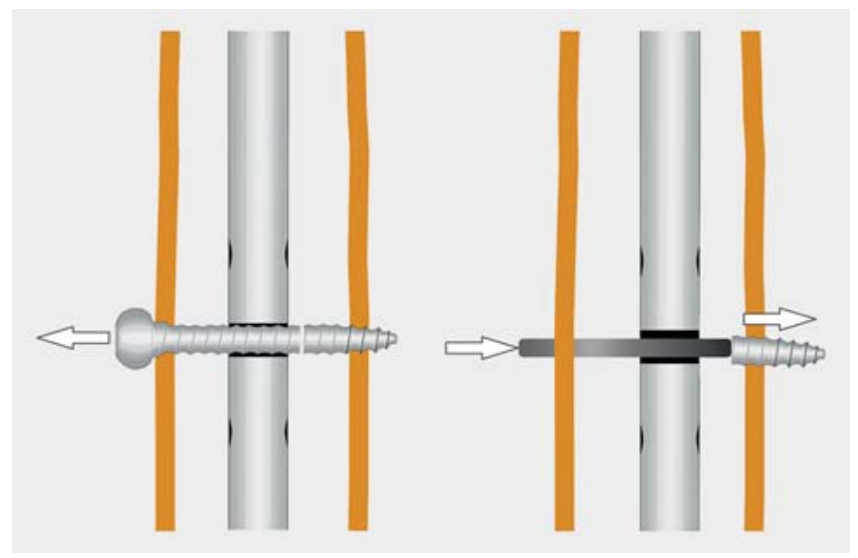

gelextraktion und sollte daher ebenfalls entfernt werden.

Das vollständige Überfräsen mit einer Hohlfräse von der Gegenseite ist u.U. schwierig und sollte auch möglichst vermieden werden, da dies den Knochendefekt vergrößert und den Halt zukünftiger Verriegelungsschrauben an dieser Stelle gefährdet.

Als mögliche Alternative kommt das Ausschlagen des Schraubenfragments zur Gegenseite mittels eines 3,8-mmKirschner-Drahtes oder eines speziellen Ausschlaginstruments infrage [8]. Aufgrund der Führung im Verriegelungsloch des Nagels weicht der Draht i.d.R. nicht
Abb. 8 Prinzip der Entfernung gebrochener Verriegelungsschrauben: Das Schraubenkopffragment wird konventionell mit dem Schraubendreher herausgedreht. Das Schraubenspitzenfragment kann dann meist ohne Überfräsung durch die Gegenkortikalis hindurch gestößelt werden.

tels eines verklemmten Olivendrahts. Die schonende Entfernung solider Nagelfragmente, wie auch die Entfernung von Tragelementen und defekten Verriegelungsschrauben, gelingt jedoch nur mittels spezieller Entfernungsinstrumentarien, welche insbesondere Hohlfräsen und linksausdrehende Außen- und Innengewinde in fein abgestuften Größen mit und ohne Stielverlängerung enthalten. Die Kenntnis des zu explantierenden Materials und der verfügbaren Methoden sowie das beschriebene Spezialinstrumentarium bilden die Voraussetzung für den Erfolg des Eingriffs.

\section{Literatur}

${ }^{1}$ Apivatthakakul T, Chiewchantanakit S. Percutaneous removal of a bent intramedullary nail. Injury 2001; 32: 725-726

2 Cruz-Sanchéz M, Torres-Claramunt R, AlierFabrego $A$ et al. Salvage for nail breakage in femoral intramedullary nailing. Injury 2015; 46: 729-733

3 Giannoudis PV, Matthews SJ, Smith RM. Removal of the retained fragment of broken solid nails by the intra-medullary route. Injury 2001, 32: 407-410

${ }^{4}$ Hahn D, Bradbury $N$, Hartley $R$ et al. Intramedullary nail breakage in distal fractures of the tibia. Injury 1996; 27: 323-327

${ }^{5}$ Hora K, Vorderwinkler KP, Vecsei $V$ et al. [Intramedullary nail removal in the upper and lower limbs. Should we recommend this operation?]. Unfallchirurg 2008; 111: 599601, 603-605

${ }^{6}$ Krettek C, Müller C, Meller R et al. [Is routine implant removal after trauma surgery sensible?]. Unfallchirurg 2012; 115: 315-322

${ }^{7}$ Küntscher G. Zur Frage der Implantatentfernung. Chirurg 1971, 42: 85-87

8 Sancineto CF, Rubel IF, Seligson D et al. Technique for removal of broken interlocking screws. J Orthop Trauma 2001; 15: 132-134

${ }^{9}$ Whalley H, Thomas G, Hull P et al. Surgeon versus metalwork-tips to remove a retained intramedullary nail fragment. Injury 2009; 40: 783-789

10 Wierer M, Biberthaler P, Mutschler $W$ et al. [Removal of a bent intramedullary tibia nail. Case report and review of literature]. Unfallchirurg 2011; 114: 629-633

hen axialen Drur Schraubenfragment (Abb. 8).

\section{Fazit}

Der Bruch von Marknägeln ist relativ selten, die Entfernung der Implantatfragmente kann aber eine Herausforderung darstellen. Zusätzlich zum Nagelbruch können weitere Umstände die Implantatentfernung erschweren, etwa der Bruch von Verriegelungsschrauben oder Trageelementen bei proximalen Femurnägeln. Für die Entfernung von Hohlnägeln sind verschiedene Methoden mit gängigen Instrumentarien beschrieben, gebräuchlich ist insbesondere die Extraktion mit-

\section{Priv.-Doz. Dr. med. Roland Biber Oberarzt \\ Univ.-Prof. Dr. med. Hermann Josef Bail Leitender Arzt}

Universitätsklinik für Orthopädie und Unfallchirurgie

Klinikum Nürnberg - Paracelsus

Medizinische Privatuniversität

Breslauer Str. 201

90471 Nürnberg

biber@klinikum-nuernberg.de 